

\title{
Analysis of Periodic Orbits with Smaller Primary As Oblate Spheroid
}

\author{
Niraj Pathak \\ Department of Mathematics, \\ Dharmsinh Desai University, Nadiad, Gujarat, 3870001, India. \\ Email address: niraj23481@yahoo.com \\ V.O.Thomas \\ Department of Mathematics,The Maharaja Sayajirao University of Baroda, \\ Vadodara, Gujarat,390002 India.
}

\begin{abstract}
We have studied closed periodic orbits with loops for two systems - Sun - Mars and Sun Earth systems - using Poincare surface section (PSS) technique. Perturbation due to oblateness for the second primary (Mars or Earth) is taken in to consideration and obtained orbits with loops varying from one to five around both primaries. It is found that the oblateness coefficient $A_{2}$ and Jacobi constant $C$ has non- negligible effect on the position of the orbits. The model may be useful for designing space mission for low - energy trajectories.

Keywords - restricted three body problem; low energy trajectory design; periodic orbits; oblateness; Poincare surface of section
\end{abstract}

\section{INTRODUCTION}

The 'low-energy' transfer trajectory is used to describe the space trajectories that consume less fuel compared to Hohman transfer (Hohmann, 1925). A Hohmann transfer is an orbital maneuver that transfers a satellite or spacecraft between two coplanar circular orbits sharing a common focus. The transfer is through an elliptical orbit which is tangent to both circular orbits. The periapse and apoapse of the transfer ellipse are the radii of the initial and final circular orbits. The classical approach of Hohmann transfer needs considerable amount of fuel for space missions. So, the space missions become costly in terms of fuel. So, missions in which trajectory can be achieved using gravity only, is more desirable in terms of cost.

The motion of an infinitesimal particle in the plane of motion of two massive point masses , called the primaries, moving about their centre of mass in a circular orbit under their mutual gravitational attraction is referred to as planar restricted three body problem (PRTBP).The masses of primary bodies are taken as $m_{1}$ and $m_{2}$ with $\quad m_{1}>m_{2}$. 
The coordinates of the bigger and smaller primaries are, respectively, $(1-\mu, 0)$ and $(\mu, 0)$, where $\mu$ denotes the number $\frac{m_{2}}{m_{1}+m_{2}}$.

[1] is fundamental book on the RTBP. Various aspects of PRTBP using Poincare surface of section (PSS) method have been discussed by a number of researchers [2], [3], [4], [5] and [6]. Periodic orbits of spacecraft around two primary bodies are required to construct low energy trajectory. [7] established three classes with orbits around both primaries depending on motion of spacecraft is prograde or retrograde in the rotating system as well as fixed system. In this paper we analyzed periodic orbits around both primaries with retrograde motion in rotating system. This family of orbit is orbit with different number of loops. In this paper we have analyzed periodic orbits having number of loops from 1 to 5 for different pair of oblateness coefficient $A_{2}$ and Jacobi constant $C$ for Sun- Mars and Sun - Earth system. It has been found that $A_{2}$ and $C$ has non-negligible effect on the position of the orbits. Irrespective of the number of loops, the second primary is always lies inside one of the loops and infinitesimal body orbits both the primaries. The closest approach of the secondary body to the second secondary body occurs for the orbit with a single loop. Therefore, such orbits can be used as low-energy orbits space mission design in solar system.

\section{II.EQUATION OF MOTION}

The perturbed mean motion $n$ of second primary body is given by,

$$
n^{2}=1+\frac{3}{2} A_{2}
$$

where

$$
A_{2}=\frac{\rho_{e}^{2}-\rho_{p}^{2}}{5 R^{2}}
$$

Here $A_{2}$ is oblateness coefficient of second primary. $\rho_{\mathrm{e}}$ and $\rho_{\mathrm{p}}$ represent equatorial and polar radii of second primary and $R$ is the distance between two primaries. The unit of mass is chosen equal to the sum of the primary masses and the unit of length is equal to their separation. The unit of time is such that the Gaussian constant of gravitation is unity in the unperturbed case. In the usual dimensionless synodic coordinate system, the equations of motion of the spacecraft are given by [8]

$$
\ddot{x}-2 n \dot{y}=\frac{\partial \Omega}{\partial x}, \quad \ddot{y}+2 n \dot{x}=\frac{\partial \Omega}{\partial y} .
$$

where

$$
\Omega=\frac{n^{2}}{2}\left[(1-\mu) r_{1}^{2}+\mu r_{2}^{2}\right]+\frac{(1-\mu)}{r_{1}}+\frac{\mu}{r_{2}}+\frac{\mu A_{2}}{2 r_{2}^{3}} \text {. }
$$

Here $\quad r_{1}^{2}=(x-\mu)^{2}+y^{2}$,

$$
\text { And } \quad r_{2}^{2}=(x+1-\mu)^{2}+y^{2} \text {. }
$$

Equations (3) and (4) lead to the first integral

$$
\dot{x}^{2}+\dot{y}^{2}=2 \Omega-C,
$$

where $C$ is Jacobi constant of integration given by

$$
C=n^{2}\left((1-\mu) r_{1}^{2}+\mu r_{2}^{2}\right)+2 \frac{(1-\mu)}{r_{1}}+2 \frac{\mu}{r_{2}}+\frac{\mu A_{2}}{r_{2}^{3}}-\dot{y}^{2} \text {. }
$$


These equations of motion are integrated in $(x, y)$ variables using a Runge- Kutta Gill fourth order method.We have constructed Poincare surface section (PSS) on the $x$, $\dot{x}$ plane. The initial values were selected along the $O x$-axis by using intervals of length 0.001 . By giving different value of $C$ we can plot the trajectories, and then analysis of orbits can be done.

\section{RESULTS AND DISCUSSON}

We shall consider two systems, the Sun - Mars system and the Sun-Earth system. For the first system we take the mass of Sun and Mars as $m_{1}=1.9881 \times 10^{30} \mathrm{~kg}$. and $m_{2}=6.4185 \times 10^{23} \mathrm{~kg}$., respectively, whereas for the second system mass of Earth is taken as $m_{2}=5.972 \times 10^{24} \mathrm{~kg}$. [3]. Thus, for the Sun - Earth and Sun- Mars systems, mass factor are 0.000003002 and 0.0000003212 respectively. Equatorial and polar radii of Earth are $6378.1 \mathrm{kms}$. and $6356.8 \mathrm{kms}$. and that of Mars are $3396.2 \mathrm{kms}$. and $3376.2 \mathrm{kms}$., respectively. The distance between Sun and Earth is taken as 149600000 kms. and distance between Sun and Mars is 227940000 kms. So, oblateness coefficient calculated from equation (2) for Sun- Earth and Sun- Mars have values $A_{2}=2.42405 \times 10^{-12}$ and $\mathrm{A}_{2}=5.21389 \times 10^{-13}$ respectively. These values of oblateness are negligible. So, in order to study the non trivial effect of oblateness on periodic orbit around both primaries, we take different values of oblateness that can make observable changes in different parameters. We have studied the effect of oblateness on the location and period of Sun- Mars system for different values of Jacobi constant C using PSS. Fig. 1 is PSS constructed for Sun Mars system when $\left(A_{2}, C\right)$ is $(0.05,2.94)$ by taking value of $x$ from $[0.7,1]$ with interval of $x$ differencing is 0.001 . Also, time span $t=10000$ and interval of time differencing is also 0.001 . So, for each $\mathrm{x}$ equations of motions are integrated using Runge -Kutta - Gill method for 1000000 times. Each solution is plotted as a point in the Fig. 1. The arcs of PSS are known as islands whose center gives periodic orbirts.

In a similar way, we can obtain PSS for Sun - Earth which is shown in Fig. 2. This PSS is also constructed for $(0.05,2.94)$. So that we can compare both PSS. Mass factor of Sun - Earth is greater than Sun - Mars.

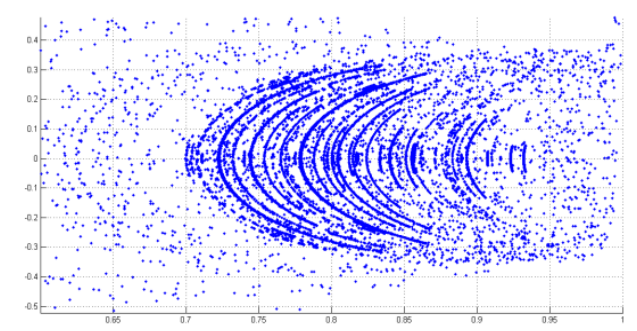

Fig. 1 PSS for $A_{2}=0.05$ and $C=2.94$, for $x=[0.7,1], t=10000$ for Sun-Mars system. 


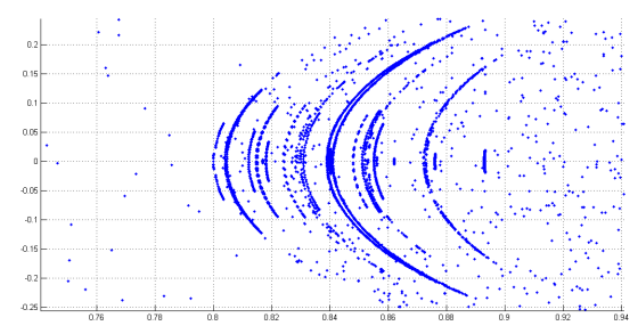

Fig. 2 PSS for $\mathrm{A}_{2}=0.05$ and $\mathrm{C}=2.94$, for $x=[0.8,1], t=10000$ for Sun - Earth system.

The numerical values of location and period of periodic orbit of spacecraft for $\mathrm{C}=2.93,2.94$, 2.95, 2.96 and for oblateness $A_{2}=0.001,0.005,0.01$ and 0.05are displayed in Table 1. It is observed from the table that a change in $\mathrm{C}$ in the range $(2.93,2.96)$ affects the location but has no effect on the period and number of loops of the orbit. Oblateness also affects the location and period of the orbit. Similarly, the effects of $\mathrm{C}$ and $\mathrm{A}_{2}$ in the location and period for the second system are studied and the numerical estimates of the changes are displayed in Table 2. It can be seen that, for both the systems, the period of orbit increases with increase in the number of loops.

A noticeable difference observed in both the systems is that for $\mathrm{C}=2.96, \mathrm{~A}_{2}=0.001$ and 0.005, single- loop periodic orbit does not exist for Sun - Earth system.

The periodic orbits starting from single - loop to five loops around the Sun - Mars system for $\mathrm{A}_{2}=0.005$ and 0.01 are shown in Figure 3(a) through Figure 3(j). It can be observed, from Figures 4 and 5, that the width of the orbit decreases continuously as the number of loops increases. Further in all cases the secondary body orbits around the second primary (Mars) in addition to orbiting both primaries. Further the secondary body is closest to Mars in the single loop closed orbit. Such orbits may be useful in the study of different aspects of both primaries. In many models available in literature not many closed orbits posses this kind of nature. We can observe similar nature in the orbits shown in Fig. 4(a) through Fig. 4(j) in the case of Sun - Earth system. Further the position of the orbit in the case of odd number of loop approaches the first primary, namely the Sun. This is true in the case of even number of loops also.

Figures 3 and 4 depict periodic orbits with number of loops referring from 1 to 5 . These orbits are given for various $\mathrm{A}_{2}$ for Sun - Mars and Sun - Earth systems. For both of these figures value of Jacobi constant $C$ is 2.93. We have studied the variation of position of periodic orbits around Sun - Mars and Sun - Earth system due to the variation in oblateness and Jacobi constants C. In Fig. 5 we have shown the variation of position of closed periodic orbit with one loop for oblateness in the range $(0,0.05)$ for Sun - Mars system corresponding to Jacobi constants $\mathrm{C}=2.93,2.94,2.95$ and 2.96. From the Fig. 5 it is clear that the position of the orbits recedes away from Mars when the oblateness increases and $\mathrm{C}$ decreases. 
Similar kind of conclusion can be drawn from Fig. 6 for the Sun - Earth system. From Figures 7 and 8, it can be observed that for given oblateness, location of periodic orbit moves away from second primary as number of loops in periodic orbit increase. Also, as oblateness increases, location of periodic orbit moves away from second primary. The variations of the eccentricity $e$ with respect to oblateness $A_{2}$ and Jacobi constant $C$ for single and two loops periodic orbits are shown in Fig. 9-12 for both Sun - Mars and Sun - Earth systems. The eccentricity decreases as $A_{2}$ increases and decreases as $C$ increases for single loop periodic orbit for both systems Sun - Mars and Sun - Earth as shown in Fig. 9 and Fig. 10. Whereas, the eccentricity increase as

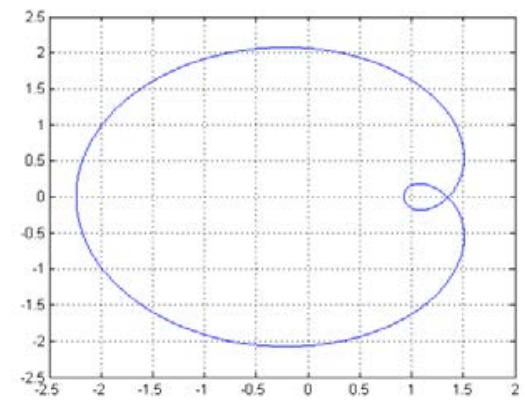

(a) $\mathrm{A}_{2}=0.005$, single loop periodic orbit.

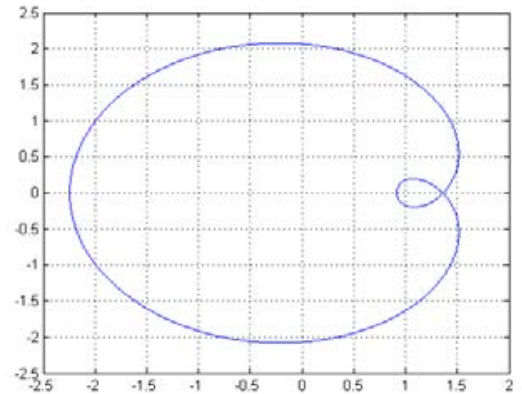

(b) $\mathrm{A}_{2}=0.01$, single loop periodic orbit.

increases and decreases as C increase for two loops periodic orbit for both systems Sun - Mars and Sun - Earth as shown in Fig. 11 and 12. The variation in eccentricity due to oblateness is exactly opposite in nature for two loops orbit in comparison to single loop orbit for Sun - Mars and Sun - Earth system.

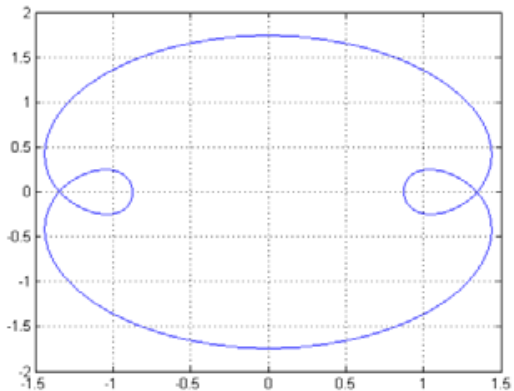

(c) $\mathrm{A}_{2}=0.005$, two loops periodic orbit.



(d) $\mathrm{A}_{2}=0.01$, two loops periodic orbit. 




(e) $\mathrm{A}_{2}=0.005$, three loops periodic orbit.

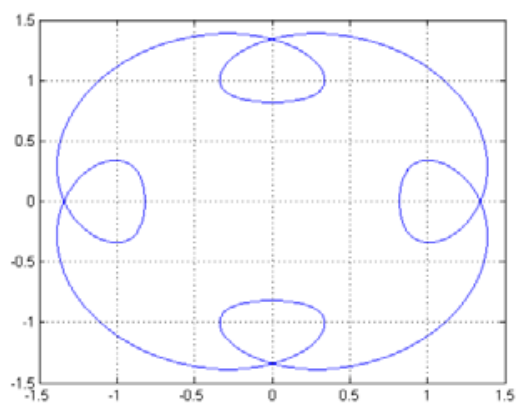

(g) $\mathrm{A}_{2}=0.005$, four loops periodic orbit.

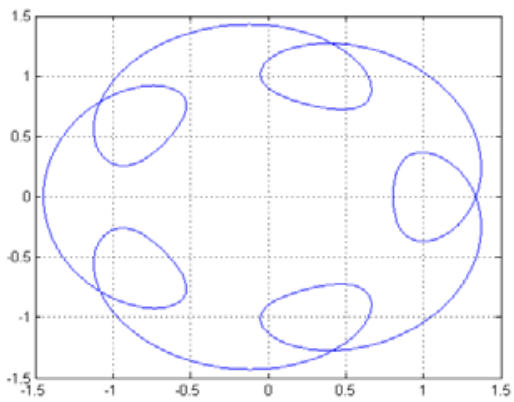

(i) $\mathrm{A}_{2}=0.005$, five loops periodic orbit.

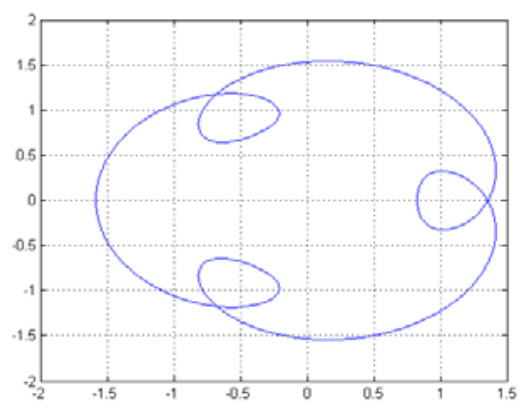

(f) $\mathrm{A}_{2}=0.01$, three loops periodic orbit.

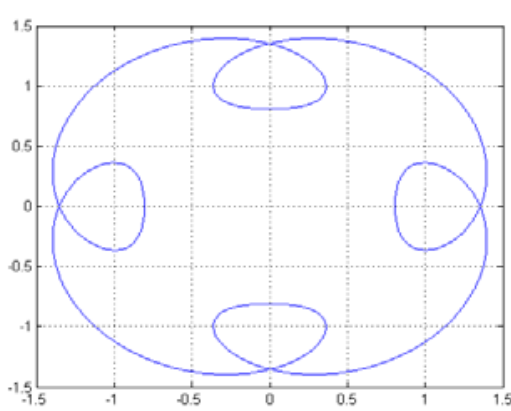

(h) $\mathrm{A}_{2}=0.01$, four loops periodic orbit.

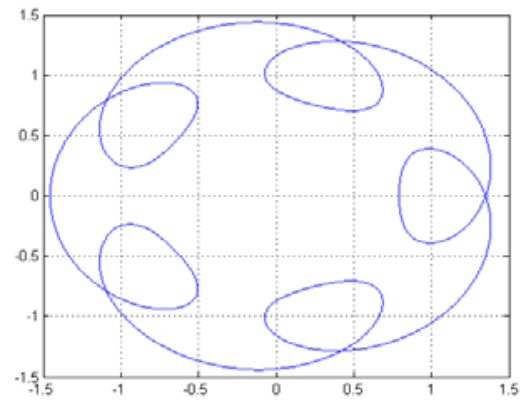

(j) $\mathrm{A}_{2}=0.01$, five loops periodic orbit.

Fig. 3 Periodic orbits around both primaries for Sun - Mars system for $\mathrm{C}=2.93$. 


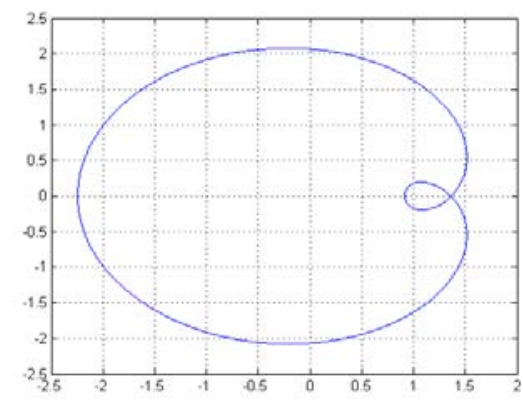

(a) $\mathrm{A}_{2}=0.005$, single loop periodic orbit.



(c) $\mathrm{A}_{2}=0.005$, two loops periodic orbit.

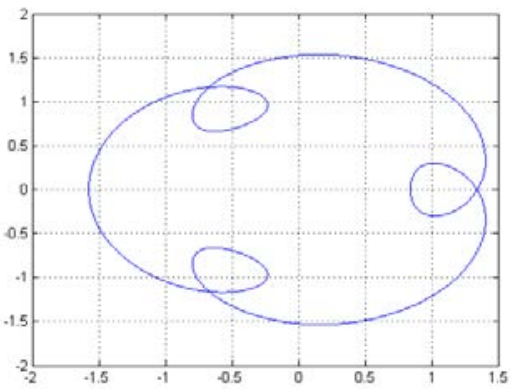

(e) $\mathrm{A}_{2}=0.005$, three loops periodic orbit.



(b) $\mathrm{A}_{2}=0.01$, single loop periodic orbit.

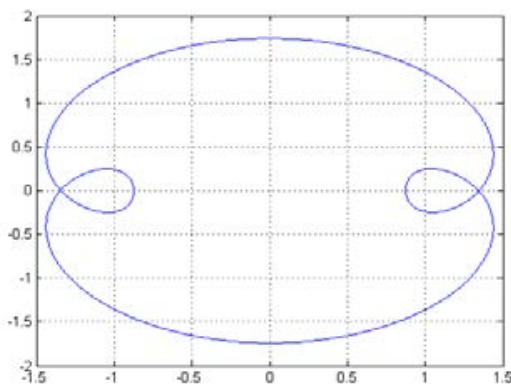

(d) $\mathrm{A}_{2}=0.01$, two loops periodic orbit.

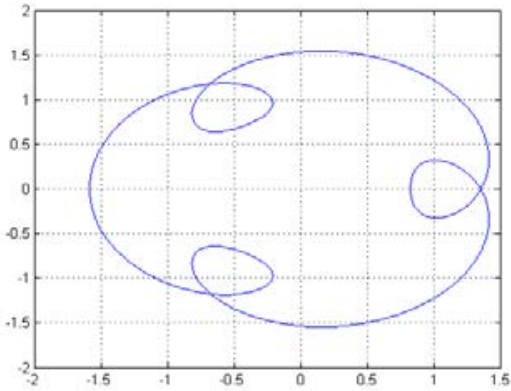

(h) $\mathrm{A}_{2}=0.01$, three loops periodic orbit. 




(g) $\mathrm{A}_{2}=0.005$, four loops periodic orbit

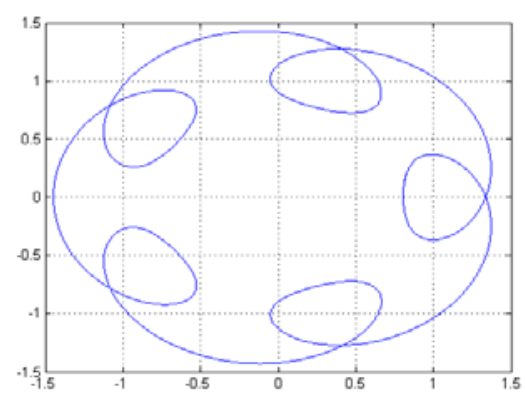

(i) $\mathrm{A}_{2}=0.005$, five loops periodic orbit.

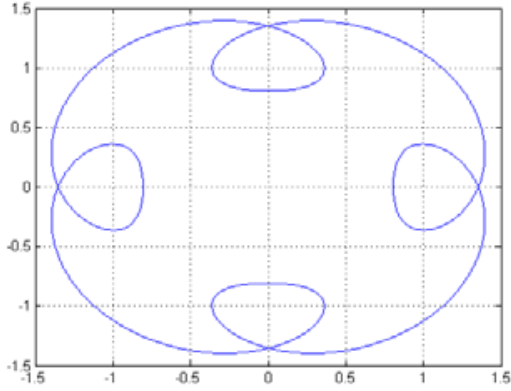

(h) $\mathrm{A}_{2}=0.01$, four loops periodic orbit.

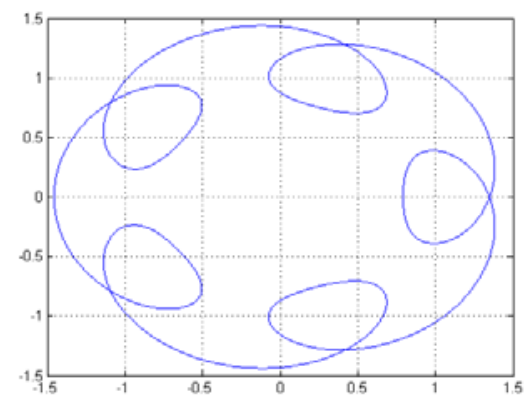

(j) $\mathrm{A}_{2}=0.01$, five loops periodic orbit.

Fig. 4 Periodic orbits around both primaries for Sun - Earth system for $\mathrm{C}=2.93$.

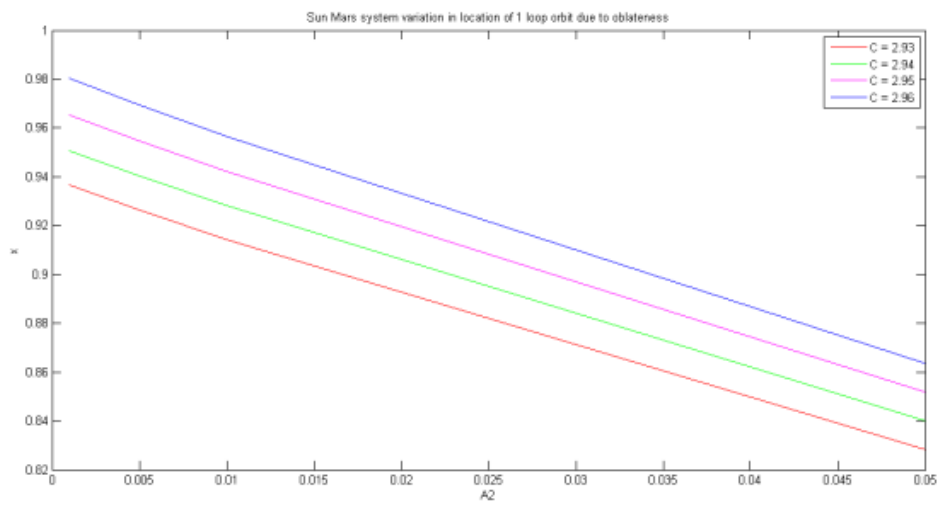

Fig. 5 Variation in location of single loop periodic orbit around Sun - Mars system due to oblateness. 
Analysis of Periodic Orbits with Smaller Primary As Oblate Spheroid Niraj Pathak, V. O. Thomas

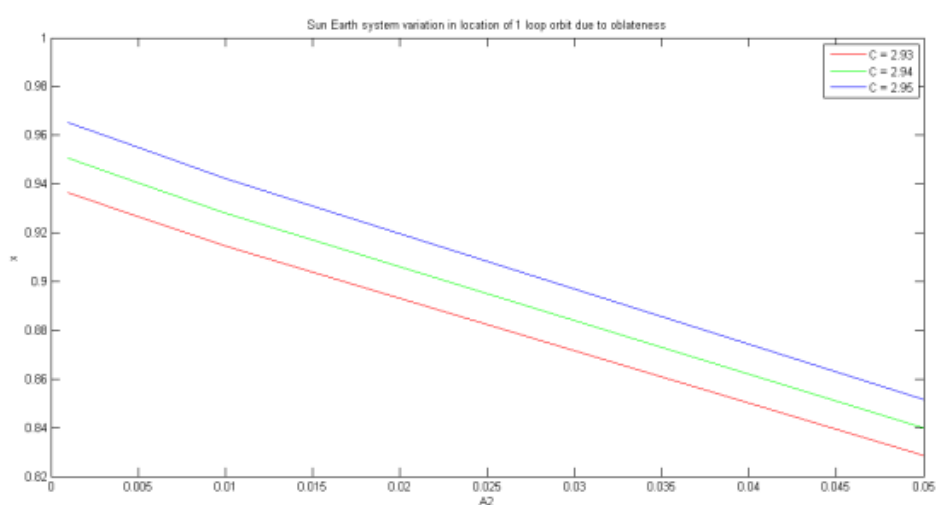

Fig. 6 Variation in location of one loop periodic orbit around Sun- Earth due to oblateness.

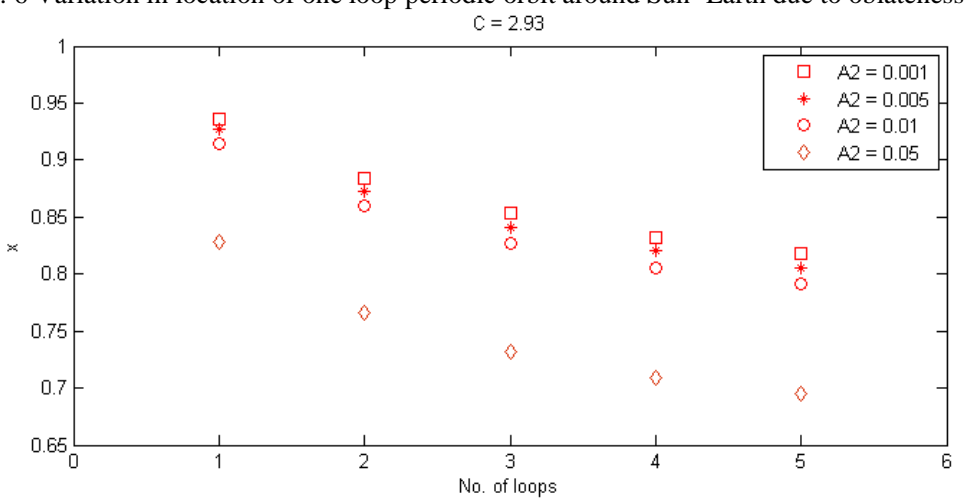

Fig. 7 Variation in location of periodic orbit of second primary around Sun and Mars for $C=2.93$ due to number of loops for different oblateness $A_{2}$.

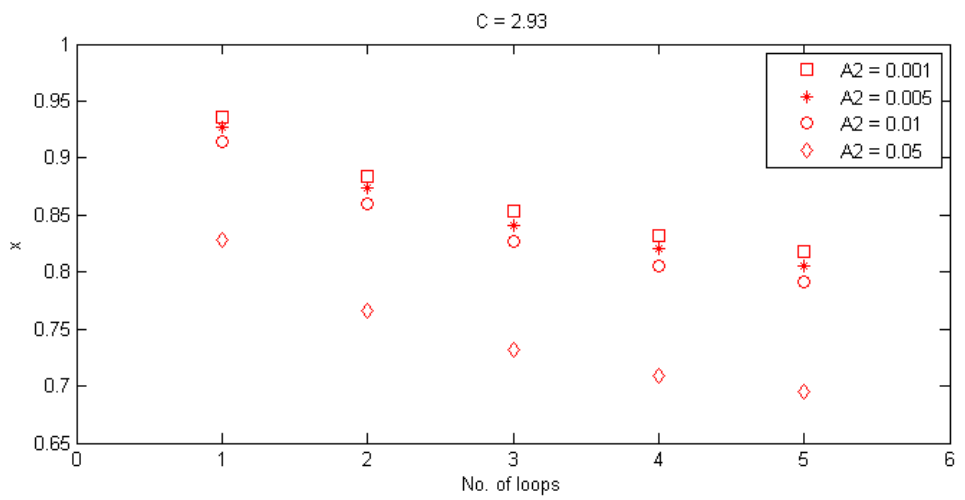

Fig. 8 Variation in location of periodic orbit of second primary around Sun - Earth system for $C=2.93$ due to number of loops for different oblateness $\mathrm{A}_{2}$. 
Analysis of Periodic Orbits with Smaller Primary As Oblate Spheroid Niraj Pathak, V. O. Thomas

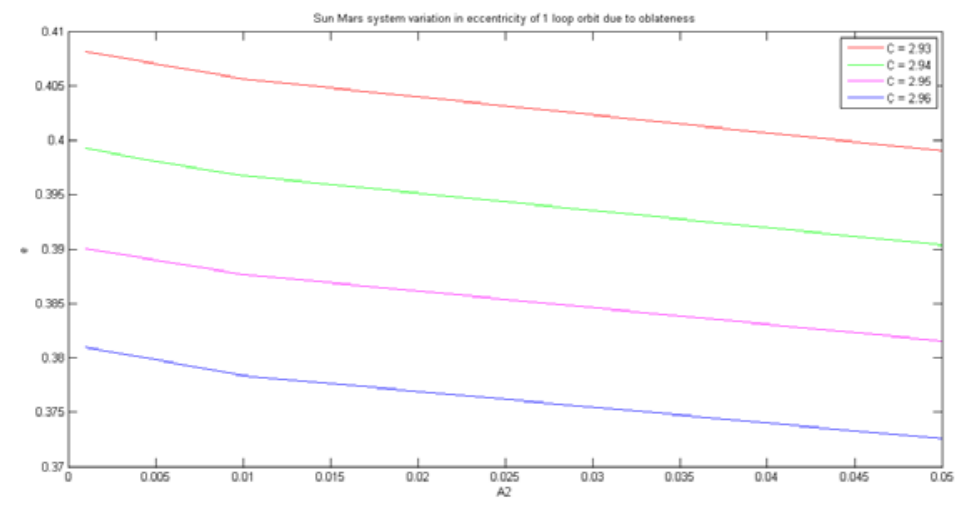

Fig. 9 Variation in eccentricity of single loop periodic orbit around Sun - Mars system due to oblateness.

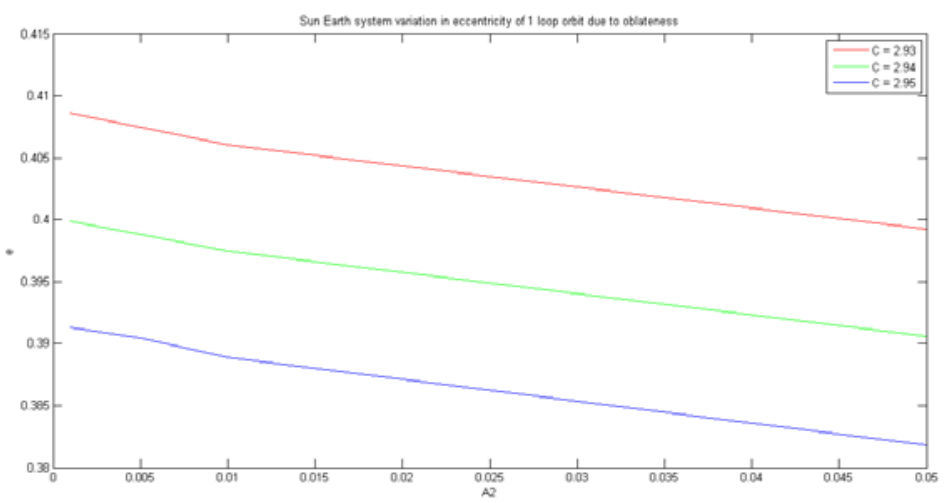

Fig. 10 Variation in eccentricity of single loop periodic orbit around Sun and Earth due to oblateness.

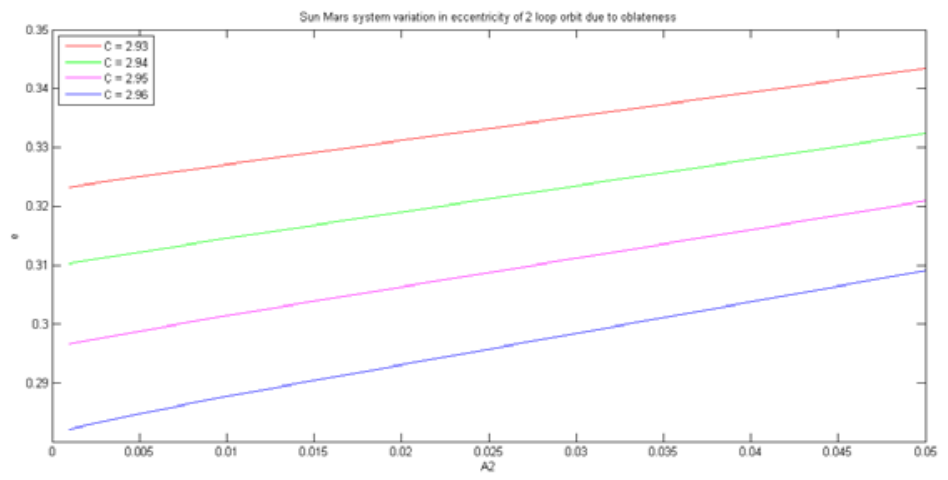

Fig. 11 Variation in eccentricity of two loops periodic orbit around Sun - Mars system due to oblateness. 


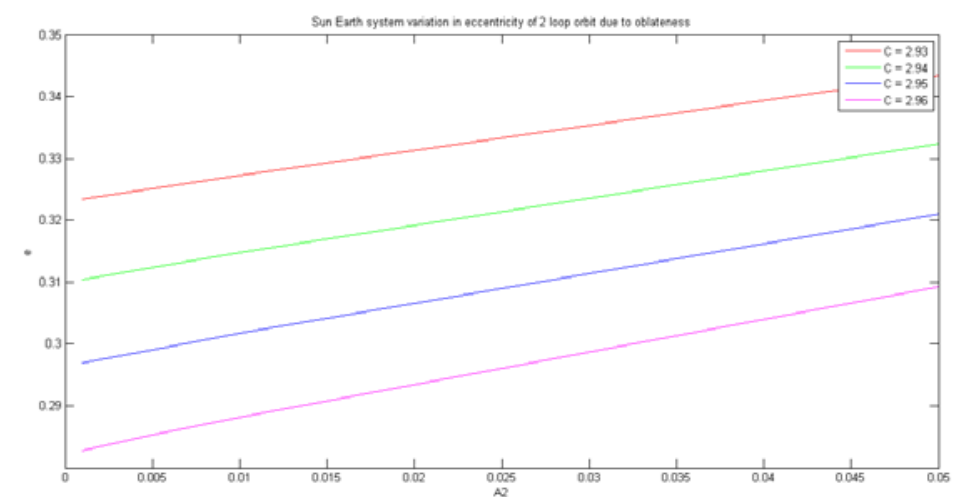

Fig. 12 Variation in eccentricity of two loops periodic orbit around Sun - Earth system due to oblateness.

\begin{tabular}{|c|c|c|c|c|c|c|c|c|c|}
\hline \multirow{2}{*}{$\begin{array}{l}\text { Number } \\
\text { of loops }\end{array}$} & \multirow[t]{2}{*}{$\mathrm{C}$} & \multicolumn{2}{|c|}{$\mathrm{A}_{2}=0.001$} & \multicolumn{2}{|c|}{$A_{2}=0.005$} & \multicolumn{2}{|c|}{$\mathrm{A}_{2}=0.01$} & \multicolumn{2}{|c|}{$\mathrm{A}_{2}=0.05$} \\
\hline & & $\begin{array}{l}\text { Location } \\
\text { (x) }\end{array}$ & $\begin{array}{c}\text { Period } \\
(\mathrm{T})\end{array}$ & $\begin{array}{c}\text { Location } \\
\text { (x) }\end{array}$ & $\begin{array}{l}\text { Period } \\
(\mathrm{T})\end{array}$ & $\begin{array}{c}\text { Location } \\
\text { (x) }\end{array}$ & $\begin{array}{l}\text { Period } \\
(\mathrm{T})\end{array}$ & $\begin{array}{l}\text { Location } \\
\text { (x) }\end{array}$ & $\begin{array}{l}\text { Period } \\
\text { (T) }\end{array}$ \\
\hline \multirow[t]{4}{*}{1} & 2.96 & 0.98028 & 13 & 0.96945 & 13 & 0.9563 & 13 & 0.86369 & 13 \\
\hline & 2.95 & 0.96525 & 13 & 0.95474 & 13 & 0.94198 & 13 & 0.85165 & 13 \\
\hline & 2.94 & 0.95068 & 13 & 0.94045 & 13 & 0.928 & 13 & 0.83988 & 13 \\
\hline & 2.93 & 0.93647 & 13 & 0.92653 & 13 & 0.9144 & 13 & 0.82835 & 13 \\
\hline \multirow[t]{4}{*}{2} & 2.96 & 0.93819 & 19 & 0.92515 & 19 & 0.90953 & 19 & 0.80504 & 19 \\
\hline & 2.95 & 0.91938 & 19 & 0.90699 & 19 & 0.8921 & 19 & 0.79159 & 19 \\
\hline & 2.94 & 0.90151 & 19 & 0.88969 & 19 & 0.87545 & 19 & 0.77857 & 19 \\
\hline & 2.93 & 0.88448 & 19 & 0.87315 & 19 & 0.85949 & 19 & 0.76593 & 19 \\
\hline \multirow[t]{4}{*}{3} & 2.96 & 0.91166 & 26 & 0.89746 & 26 & 0.88061 & 25 & 0.7715 & 25 \\
\hline & 2.95 & 0.89087 & 26 & 0.87757 & 26 & 0.86174 & 25 & 0.75762 & 25 \\
\hline & 2.94 & 0.87144 & 26 & 0.8589 & 26 & 0.84392 & 25 & 0.74425 & 25 \\
\hline & 2.93 & 0.85315 & 26 & 0.84127 & 26 & 0.82703 & 25 & 0.73133 & 25 \\
\hline \multirow[t]{4}{*}{4} & 2.96 & 0.89323 & 32 & 0.87841 & 32 & 0.86095 & 32 & 0.750015 & 31 \\
\hline & 2.95 & 0.87139 & 32 & 0.85766 & 32 & 0.84138 & 32 & 0.73601 & 31 \\
\hline & 2.94 & 0.8512 & 32 & 0.83835 & 32 & 0.82305 & 32 & 0.72254 & 31 \\
\hline & 2.93 & 0.83233 & 32 & 0.82022 & 32 & 0.80575 & 32 & 0.70957 & 31 \\
\hline \multirow[t]{4}{*}{5} & 2.96 & 0.87973 & 38 & 0.86457 & 38 & 0.8468 & 38 & 0.735165 & 37 \\
\hline & 2.95 & 0.85732 & 38 & 0.84337 & 38 & 0.82688 & 38 & 0.72113 & 37 \\
\hline & 2.94 & 0.83673 & 38 & 0.82373 & 38 & 0.8083 & 38 & 0.70767 & 37 \\
\hline & 2.93 & 0.81758 & 38 & 0.80538 & 38 & 0.79083 & 38 & 0.69471 & 37 \\
\hline
\end{tabular}


Table 2: Analysis of periodic orbit for different pairs of $A_{2}$ and $C$ for Sun - Earth system.

\begin{tabular}{|c|c|c|c|c|c|c|c|c|c|}
\hline \multirow{2}{*}{$\begin{array}{l}\text { Number } \\
\text { of loops }\end{array}$} & \multirow[t]{2}{*}{$\mathrm{C}$} & \multicolumn{2}{|c|}{$\mathrm{A}_{2}=0.001$} & \multicolumn{2}{|c|}{$\mathrm{A}_{2}=0.005$} & \multicolumn{2}{|c|}{$A_{2}=0.01$} & \multicolumn{2}{|c|}{$\mathrm{A}_{2}=0.05$} \\
\hline & & $\begin{array}{c}\text { Location } \\
(\mathrm{x})\end{array}$ & $\begin{array}{c}\text { Period } \\
(\mathrm{T})\end{array}$ & $\begin{array}{c}\text { Location } \\
(\mathrm{x})\end{array}$ & $\begin{array}{c}\text { Period } \\
(\mathrm{T})\end{array}$ & $\begin{array}{c}\text { Location } \\
\text { (x) }\end{array}$ & $\begin{array}{c}\text { Period } \\
(\mathrm{T})\end{array}$ & $\begin{array}{c}\text { Location } \\
\text { (x) }\end{array}$ & $\begin{array}{c}\text { Period } \\
(\mathrm{T})\end{array}$ \\
\hline \multirow[t]{4}{*}{1} & 2.96 & - & - & - & - & 0.9565 & 13 & 0.86375 & 13 \\
\hline & 2.95 & 0.9653 & 13 & 0.95485 & 13 & 0.9421 & 13 & 0.8517 & 13 \\
\hline & 2.94 & 0.9507 & 13 & 0.9405 & 13 & 0.9281 & 13 & 0.83994 & 13 \\
\hline & 2.93 & 0.9365 & 13 & 0.92657 & 13 & 0.91446 & 13 & 0.8284 & 13 \\
\hline \multirow[t]{4}{*}{2} & 2.96 & 0.93825 & 19 & 0.92523 & 19 & 0.90961 & 19 & 0.805085 & 19 \\
\hline & 2.95 & 0.91942 & 19 & 0.90704 & 19 & 0.89215 & 19 & 0.791635 & 19 \\
\hline & 2.94 & 0.90154 & 19 & 0.88973 & 19 & 0.8755 & 19 & 0.7786 & 19 \\
\hline & 2.93 & 0.8845 & 19 & 0.87319 & 19 & 0.85953 & 19 & 0.76596 & 19 \\
\hline \multirow[t]{4}{*}{3} & 2.96 & 0.91172 & 26 & 0.89753 & 26 & 0.88069 & 25 & 0.77155 & 25 \\
\hline & 2.95 & 0.89091 & 26 & 0.87763 & 26 & 0.86179 & 25 & 0.75766 & 25 \\
\hline & 2.94 & 0.87149 & 26 & 0.85895 & 26 & 0.84397 & 25 & 0.74428 & 25 \\
\hline & 2.93 & 0.853183 & 26 & 0.84131 & 26 & 0.82706 & 25 & 0.73136 & 25 \\
\hline \multirow[t]{4}{*}{4} & 2.96 & 0.89329 & 32 & 0.87847 & 32 & 0.86103 & 32 & 0.750055 & 31 \\
\hline & 2.95 & 0.87145 & 32 & 0.85771 & 32 & 0.84143 & 32 & 0.73605 & 31 \\
\hline & 2.94 & 0.85124 & 32 & 0.83839 & 32 & 0.82309 & 32 & 0.72258 & 31 \\
\hline & 2.93 & 0.83237 & 32 & 0.82026 & 32 & 0.80579 & 32 & 0.7096 & 31 \\
\hline \multirow[t]{4}{*}{5} & 2.96 & 0.8798 & 38 & 0.864634 & 38 & 0.84687 & 38 & 0.7352 & 37 \\
\hline & 2.95 & 0.857375 & 38 & 0.84342 & 38 & 0.82693 & 38 & 0.72117 & 37 \\
\hline & 2.94 & 0.83677 & 38 & 0.82378 & 38 & 0.80834 & 38 & 0.7077 & 37 \\
\hline & 2.93 & 0.81762 & 38 & 0.8054 & 38 & 0.79087 & 38 & 0.69474 & 37 \\
\hline
\end{tabular}

\section{CONCLUSION}

In this paper we have studied the effect of oblateness on the position closed periodic orbit with loops varying from 1 to 5 for Sun - Mars and Sun - Earth systems, respectively. A noticeable change observed between two systems is that for $C=2.96, A_{2}=0.001$ and 0.005 , single loop periodic orbit does not exist for Sun - Earth system. All closed orbits with loops varying from 1 to 5, the second primary (here Mars or Earth) is always inside one of the loops and the trajectory orbits both primaries. For both the systems, the period of orbit increases with increase in the number of loops. Also, for both the systems, for the given $\mathrm{C}$, as oblateness increases, location of periodic orbit moves towards Sun. Whereas, for the given oblateness as C increases, location of the periodic orbit moves away from the Sun. Further the eccentricity for both Sun - Mars and Sun - Earth systems decrease with increase in oblateness for single loop orbits, whereas, for orbits with loops two, the eccentricity increases with increase in oblateness. The variation in eccentricity due to oblateness is exactly opposite in nature for two loops orbit in comparison to single loop orbit for Sun - Mars and Sun - Earth system.

Thus, the present analysis of the two systems - Sun - Mars and Sun - Earth systems - using PSS technique reveals that $A_{2}$ and $C$ has non negligible effect on the position of the obit. Since the second primary body is always inside one of the loops and the secondary body orbits both the primaries, these orbits can be used for designing low - energy space mission in solar system. 
Analysis of Periodic Orbits with Smaller Primary As Oblate Spheroid Niraj Pathak, V. O. Thomas

\section{References}

1. Szebehely, V., Theory of Orbits, Academic Press, San Diego, 1967.

2. Dutt. P., and Sharma, R.K., "Analysis of Periodic and Quasi-Periodic Orbits in the Earth-Moon System,” Journal of Guidance , Control , and Dynamics, vol. 33, pp. 1010-1017, 2010.

3. Dutt. P., and Sharma, R.K., "Evolution of Periodic Orbits in the Sun-Mars System,” Journal of Guidance, Control , and Dynamics, vol. 34, pp. 635-644, 2011.

4. Safiyabeevi, A., and Sharma, R.K., "Analysis of Periodic Orbits in the Saturn-Titan System Using the Method of Poincare Section Surfaces,” Astrophysics and Space Science, vol. 333, pp. 37 - 48, 2011.

5. Dutt. P., and Sharma, R.K., "On the Evolution of the "f" Family in the Restricted Three-Body Problem," Astrophysics and Space Science, vol. 340, pp. 63-70, 2012.

6. Pathak. N., Sharma, R. K., and Thomas, V. O., “ Evolution of Periodic Orbits in the Sun-Saturn System,” International Journal of Astronomy and Astrophysics, vol. 6, pp. 175-197, 2016.

7. Stromgren, E. O. "Connaissance Actualle des Orbites dans le Problem des Trois Corps." Vol. 9, Publications and Minor Communications of Copenhagen Observatory, Publication 100, Copenhagen University, Astronomical Observatory, Denmark, vol. 87, 1935.

8. Sharma, R.K., " The Linear Stability of Libration Points of the Photogravitational Restricted Three-Body Problem When the Smaller Primary Is an Oblate Spheroid.”, Astrophysics and Space Science , vol. 135, pp. 271-281, 1987. 